\title{
Derivation and Solution of a New Three-Dimensional Heat Conduction Model
}

\author{
Yuanjuan Yang \\ North China Electric Power University, Baoding, Hebei Province, China \\ 714827652@qq.com
}

Keywords: Heat conduction equation; Integral transform; Finite volume method; MATLAB

\begin{abstract}
This paper proposes a new three-dimensional heat conduction model, which is based on the basic method for solving the one-dimensional heat conduction equation and the finite volume method to solve two-dimensional heat conduction equation, integrating the basic fact of three-dimensional heat conduction. Also, we presented the solving method of the new modle. The feasibility of this method is proved by the method of graphic display, which is based on the PDE toolbox in MATLAB.
\end{abstract}

\section{Introduction}

First of all, we analyzed a series of thermal infrared Fig. 1 [1], which illustrate adding hot water to warm water. In this set of images, we can see clearly when we pour into hot water along the wall of the cup, heat will first be conducted along the wall of the cup. After it arrives at the bottom of the cup, the transmission along the bottom plane. When the bottom heat reaches a certain value, the heat from the bottom of the cup will slowly upward, and the heat of water injection point will begin to carry out the two-dimensional heat conduction on the water surface at the same time. At last, the heat from the bottom of the cup continues conducting upward, while the heat on the surface of the water is conducted down, and then the whole glass of water tends to be a heat balance gradually.
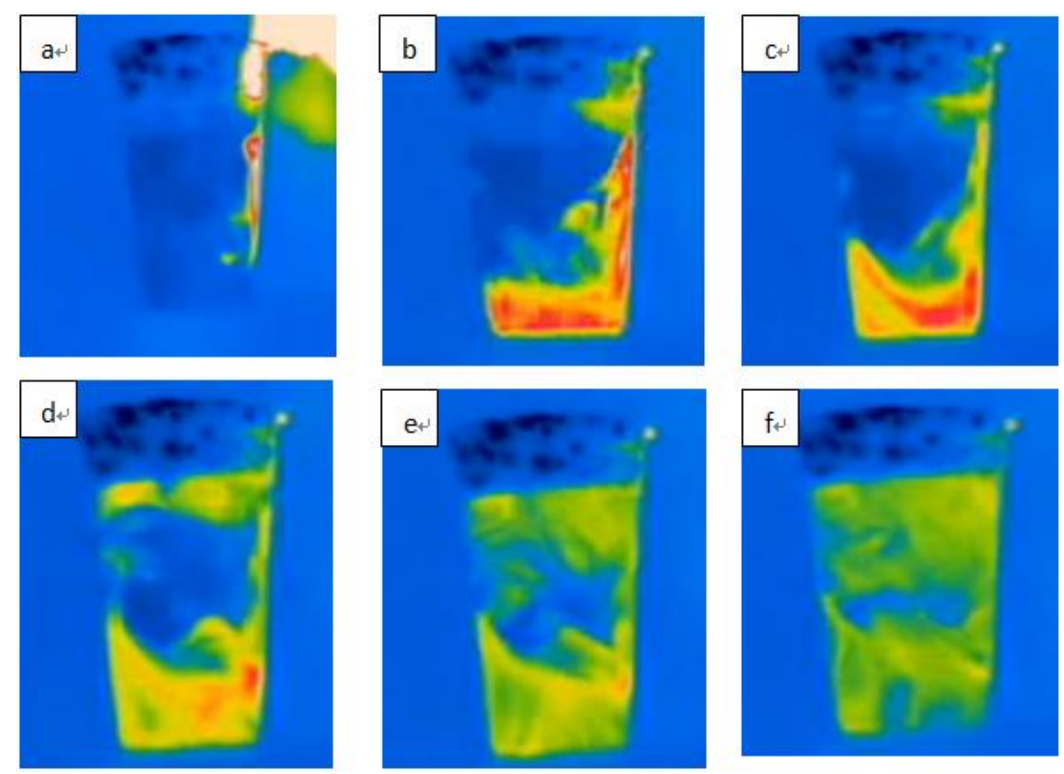

Figure 1. A series of thermal infrared images

The right upper corner of the picture with a high rate of exposure is a cup of hot water. The middle of the picture is a cup of cold water, and the hot water is poured into the cold water slowly.

a. The right upper corner of the picture with a high rate of exposure is a cup of hot water. The middle of the picture is a cup of cold water, and the hot water is poured into the cold water slowly.

b. Hot water has been poured into the cold water, most of the heat concentrated on the bottom 
of the cup

c. The cup bottom heat has already completed the two-dimensional conduction and begin the one-dimensional conduction upward, while the heat accumulation on the surface of the water begin the two-dimensional conduction

d. The heat on the surface of the water also completed the two-dimensional conduction and begin to do the downward one -dimensional conduction

e. Complex mixed conduction

f. Heats tends to be balanced

The one-dimensional conduction and two-dimensional conduction mentioned above is only the main conduction mode at the time. In fact, in the whole process, both one dimensional and two dimensional are conducting continuously

Based on the fact of the three-dimensional heat conduction, we present our model: when adding hot water into a container, the heat go through a conduction process as mentioned above, and that ultimately tend into equilibrium. We decompose the three-dimensional heat conduction process into two stages: one is two-dimensional heat conduction in the horizontal direction, the other is one-dimensional heat conduction in the vertical direction. Then going on the analysis separately.

One-Dimensional Heat Conduction in the Vertical Direction. [2]

$$
\frac{\partial u}{\partial t}=\alpha^{2} \frac{\partial^{2} \mathbf{u}}{\partial \mathbf{z}^{2}}(\mathrm{t}>0,0<z<h \mathrm{~m})
$$

Two-Dimensional Heat Conduction in the Horizontal Direction. [3]

$$
\frac{\partial \mathrm{u}}{\partial \mathrm{t}}=\alpha^{2}\left(\frac{\partial^{2} \mathrm{u}}{\partial \mathrm{x}^{2}}+\frac{\partial^{2} \mathrm{u}}{\partial \mathrm{y}^{2}}\right)
$$

One dimensional heat conduction and two dimensional heat conduction alternation and finally the whole container reach the heat balance.

\section{Solution of Three Dimensional Heat Conduction Model}

Solution of One Dimensional Heat Conduction Equation. Do Fourier transform to function u and $\delta$ of $\mathrm{z}$ :

$$
\begin{aligned}
& \mathrm{u}(0, \mathrm{z})=\delta(\mathrm{z}) \\
& \overline{\mathrm{u}}(\mathrm{t}, \lambda)=\mathrm{F}[\mathrm{u}(\mathrm{t}, \mathrm{z})]=\int_{-\infty}^{+\infty} \mathrm{u}(\mathrm{t}, \xi) \mathrm{e}^{\mathrm{i} \lambda \xi} \mathrm{d} \xi
\end{aligned}
$$

When $z \rightarrow h_{m}, u \rightarrow 0$, according to the properties of Fourier transform, we can get the initial problem on ordinary differential equation as below:

$$
\begin{aligned}
& \frac{\mathrm{d} \overline{\mathrm{u}}}{\mathrm{dt}}=\alpha^{2}\left[(-\mathrm{i} \lambda)^{2} \overline{\mathrm{u}}=-\alpha^{2} \mathrm{z}^{2} \overline{\mathrm{u}}\right] \\
& \left.\overline{\mathrm{u}}\right|_{\mathrm{t}=0}=1
\end{aligned}
$$

And the answer is as follows:

$$
\overline{\mathrm{u}}(\mathrm{t}, \lambda)=\mathrm{e}^{-\alpha^{2} \mathrm{z}^{2} \mathrm{t}}
$$

We do Fourier transform, and get the basic solution to the Cauchy problem of one dimensional heat conduction equation.

$$
u(t, z)=\frac{1}{2 \pi} \int_{-\infty}^{+\infty} \bar{u} e^{-i \lambda z} d \lambda=\frac{1}{2 \pi} \int_{-\infty}^{+\infty} e^{-\alpha^{2} z^{2} t-i \lambda z} d \lambda=\frac{1}{2 \alpha \sqrt{\pi t}} e^{-\frac{z^{2}}{4 \alpha^{2} t}}
$$

As for the basic solution of one dimensional heat conduction equation, by using the temperature distribution function $\mathrm{u}(\mathrm{t}, \mathrm{z})$ of different time $\mathrm{t} 1<\mathrm{t} 2<\mathrm{t} 3$, draw the $\mathrm{u}-\mathrm{z}$ curve, as shown in Fig. 2 .

From Fig. 2, the smaller the $t$, the higher the curve in the vicinity of 0 is; the larger the $t$, the flatter the curve is. When the $t->\infty$, the temperature at each point tends to zero; when $t->0$, the 
temperature distribution tends to $\delta(\mathrm{x})$. However, regardless of the value of the $\mathrm{T}$, the sum of the area under the curve is always 1 , which satisfies the normalization condition.

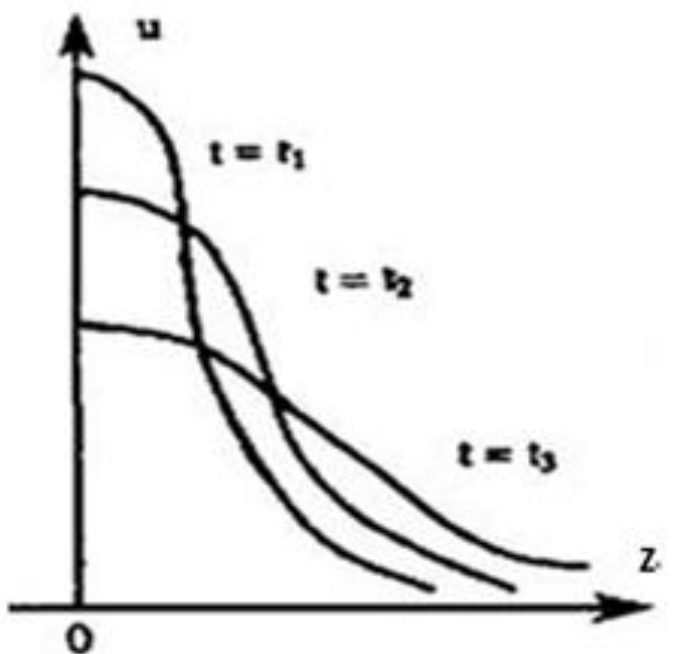

Figure 2. u-z curve

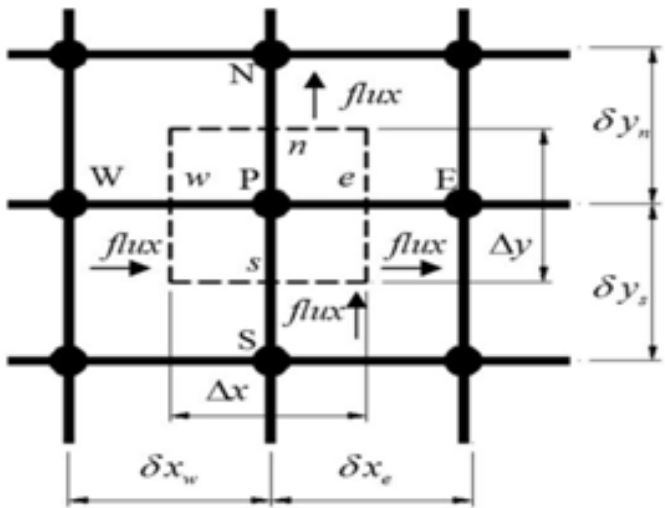

Figure 3. Grid cells and nodes

Solution of Two Dimensional Heat Conduction Equation. Finite Volume Method. Finite volume method is a kind of numerical calculation method, which by using algebraic equations to replace the original heat transfer partial differential equation that is difficult to solve directly. It is similar to finite difference method and finite element method, both of them are solving the discrete element grid node value thus to obtain a solution of heat transfer problems. As shown in Fig. 3, as for the two-dimensional problems, "finite volume unit 'refers to a closed area which centring on node P and Formed by the East (E) and West (W), South (S), North (N) interface(the dotted box in Fig. 3 ).The adjacent volume elements transfer the heat flow through the interface (Fig. 3 flux). The inflow heat from a finite volume unit is equal to the outflow heat from the neighboring finite volume unit. So it is a kind of energy conservation calculation method, and the finite volume method is widely used in the numerical solution for heat and momentum transfer.

Partial Differential Equations for Two Dimensional Heat Transfer Problem.

$$
\frac{\partial \mathrm{u}}{\partial \mathrm{t}}=\alpha^{2}\left(\frac{\partial^{2} \mathrm{u}}{\partial \mathrm{x}^{2}}+\frac{\partial^{2} \mathrm{u}}{\partial \mathrm{y}^{2}}\right)
$$

It can be transformed as:

$$
\rho c \frac{\partial \mathrm{T}}{\partial \mathrm{t}}=\frac{\partial}{\partial \mathrm{x}}\left(\lambda \frac{\partial \mathrm{T}}{\partial \mathrm{x}}\right)+\frac{\partial}{\partial \mathrm{y}}\left(\lambda \frac{\partial \mathrm{T}}{\partial \mathrm{y}}\right)+\mathrm{S}
$$

$\mathrm{x}$ and $\mathrm{y}$, respectively, show the horizontal and vertical space coordinates;

$\rho, c, t, T, \lambda, S$, respectively, which indicated that density, heat capacity, time, temperature, thermal conduction coefficient, energy item.

Finite volume element integral as follows:

$$
\int_{\tau}^{\tau+\Delta \tau} \int_{\omega}^{\varepsilon} \int_{S}^{n} \rho c \frac{\partial T}{\partial \tau} d x d y=\int_{\tau}^{\tau+\Delta \tau} \int_{\omega}^{\varepsilon} \int_{S}^{n}\left(\frac{\partial}{\partial x}\left(\lambda \frac{\partial T}{\partial x}\right)+\frac{\partial}{\partial y}\left(\lambda \frac{\partial T}{\partial y}\right)+S\right) d x d y d \tau
$$

The equivalent discrete algebraic equations of the two-dimensional heat transfer partial differential equations can be derived from the above integral equations.

$$
\begin{aligned}
& a_{P} T_{P}=a_{E} T_{E}+a_{W} T_{W}+a_{N} T_{N}+a_{S} T_{S}+S_{u} \Delta V+a_{P}^{0} T_{P}^{0} \\
& a_{P}=a_{E}+a_{W}+a_{N}+a_{S}+a_{P}^{0}-S_{P} \Delta V \\
& a_{P}^{0}=\frac{\rho c \Delta V}{\Delta \tau}
\end{aligned}
$$




$$
\begin{aligned}
& \mathrm{a}_{\mathrm{E}}=\lambda_{\mathrm{e}} \frac{\Delta \mathrm{y}}{\delta \mathrm{x}_{\mathrm{e}}}, \mathrm{a}_{\mathrm{W}}=\lambda_{\mathrm{w}} \frac{\Delta \mathrm{y}}{\delta \mathrm{x}_{\mathrm{w}}}, \mathrm{a}_{\mathrm{N}}=\lambda_{\mathrm{n}} \frac{\Delta \mathrm{y}}{\delta \mathrm{x}_{\mathrm{n}}}, \mathrm{a}_{\mathrm{S}}=\lambda_{\mathrm{s}} \frac{\Delta \mathrm{y}}{\delta \mathrm{x}_{\mathrm{s}}} \\
& \Delta \mathrm{V}=\Delta \mathrm{x} \Delta \mathrm{y}
\end{aligned}
$$

Boundary Conditions and Initial Conditions. Numerical solution of heat transfer problem can be solved by MATLAB under certain boundary conditions and initial conditions.

\section{Initial Conditions.}

$(x, y, z) \in G G$ is the maximum capacity of the container $\mathrm{u}(\mathrm{x}, \mathrm{y}, \mathrm{z})=\mathrm{u}_{0} u_{0}$ is initial temperature

\section{Boundary Conditions. [4]}

$\Gamma=\partial \mathrm{G}$.
1) $\left.\quad \mathrm{u}\right|_{\Gamma}=\mathrm{g}(\mathrm{x}, \mathrm{y}, \mathrm{z}, \mathrm{t})(\mathrm{x}, \mathrm{y}, \mathrm{z}) \in \Gamma, \mathrm{t}>0$
(Dirichlet boundary conditions)
2) $\left.\quad \mathrm{k} \frac{\partial \mathrm{u}}{\partial \mathrm{n}}\right|_{\Gamma}=\mathrm{g}(\mathrm{x}, \mathrm{y}, \mathrm{z}, \mathrm{t})(\mathrm{x}, \mathrm{y}, \mathrm{z}) \in \Gamma, \mathrm{t}>0$
3) $\left.\left(\frac{\partial \mathrm{u}}{\partial \mathrm{n}}+\alpha \mathrm{u}\right)\right|_{\Gamma}=\mathrm{g}(\mathrm{x}, \mathrm{y}, \mathrm{z}, \mathrm{t})(\mathrm{x}, \mathrm{y}, \mathrm{z}) \in \Gamma, \mathrm{t}>0$
(D-N mixed boundary conditions)

\section{Graphic Description}

Finally, we obtain the heat transfer process in the profile and the cross section of a bathtub by the PDE toolbox in MATLAB.

In the process of problem solving, we find that time $t$ is a very important parameter. It is necessary for us to study the details of the changes in heat conduction over time. What's more, the effective description of the spatial location is also a key to solve the problem of three-dimensional heat conduction.

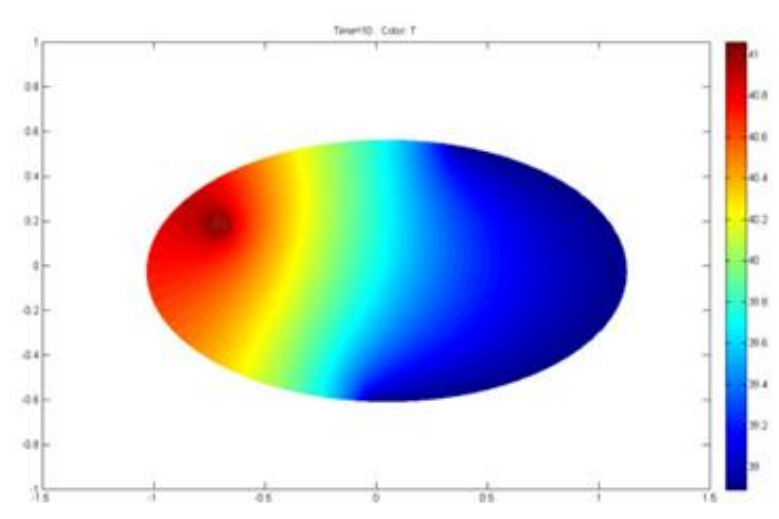

Figure 4. Temperature field of cross section

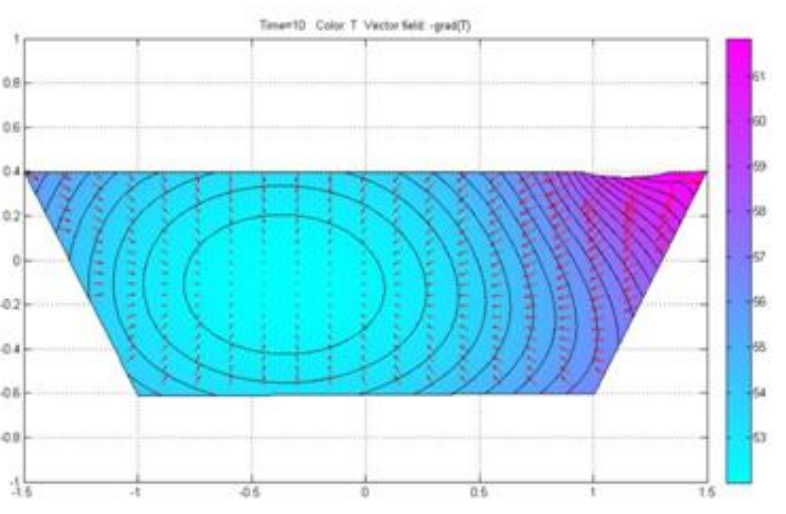

Figure 5. Temperature field distribution of longitudinal section

In Fig. 4, we use the ellipse to represent the cross-section of the container, and color is used to represent the heat level----red indicates high temperature, blue means low temperature. The legend on the right clearly expressed the color change of heat from high to low, which vividly describes the two-dimensional heat conduction in the horizontal plane.

In Fig. 5, we use a ladder to simply shows the container longitudinal profile, and still use color indicate heat level. At the same time, drawing the direction of heat conduction and the isotherm in this state, which describe the deep meaning of our three-dimensional conduction model specifically, by the PDE toolbox.

\section{Conclusion}

The above three-dimensional heat conduction model and its solution process are highly consistent 
with the true fact. Using MATLAB to achieve the results of the heat conduction process simulation is also feasible and reliable. These show that the above model is of universal value in solving the three-dimensional heat conduction problem.

\section{References}

[1] http://www.tudou.com/programs/view/Z9jxHSaXk3A/

[2] G. Cao, G.Z. Wang and X.R. Ren. Basic solution of one dimensional heat conduction equation [J]. Journal of Shandong University of technology, 2005, 19 (4): 77-80. (In Chinese)

[3] Q. Xue and X.F. Xiao. Two dimensional heat conduction equation with finite volume method MATLAB computer engineering and applications, [J]. 2012, 48 (24): 197-200. (In Chinese)

[4] http://wenku.baidu.com/link?url=ckLzvPep5_I-xapNAPm0LVxfrv47Zf69ELgdn10A-9bZ9Kk5 fkZuvUNbXUtTvutgVKTgCKNWf9AGe6FepsRn5TSYBKiSCMz4goiwkPWRpdO ） (In Chinese)

[5] H.L. Sun A high accuracy explicit scheme for solving three dimensional heat conduction equations [J]. Applied Mathematics and mechanics, 1999, 20 (7): 737-742. (In Chinese)

[6] W.G. Zhou Three dimensional heat conduction equation modified local C-N method [J]. Journal of Shandong University of Technology (NATURAL SCIENCE EDITION), 2015 (1): 10-14. (In Chinese)

[7] Z.L. Wang, C. Gong and Q. He Proficient in MATLAB scientific computing [M]. Machinery Industry Press, 2012 (In Chinese)

[8] W.P. Zeng A family of three dimensional heat conduction equation in a family of two layer explicit schemes [J]. Applied Mathematics and mechanics, 2000, 21 (9): 966-972. (In Chinese)

[9] J.L. Xu and B.S. Tang. Numerical calculation of one dimensional heat conduction equation [J]. Journal of Huaiyin Normal University (NATURAL SCIENCE EDITION), 2004, 3 (3): 210-214. (In Chinese)

[10] W.S. Li, W.Q. Wang. A finite difference domain decomposition algorithm for two-dimensional heat conduction equation [J]. Journal of Shandong University (SCIENCE EDITION), 2011, 46 (12): 1-5. (In Chinese) 